\title{
Biomarker Localization, Analysis, Visualization, Extraction, and Registration (BLAzER) Methodology for Research and Clinical Brain PET Applications
}

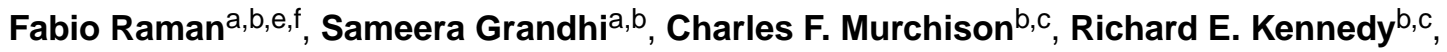 \\ Susan Landau ${ }^{d}$, Erik D. Roberson ${ }^{b, e, f}$, Jonathan McConathy ${ }^{a}, b,{ }^{,}$, Alzheimer's Disease \\ Neuroimaging Initiative ${ }^{1}$ \\ aDepartment of Radiology, University of Alabama at Birmingham, Birmingham, AL, USA \\ ${ }^{b}$ Alzheimer's Disease Center, University of Alabama at Birmingham, Birmingham, AL, USA \\ 'Department of Biostatistics, University of Alabama at Birmingham, Birmingham, AL, USA \\ dHelen Wills Neuroscience Institute, University of California at Berkeley, Berkeley, CA, USA \\ eDepartment of Neurology, University of Alabama at Birmingham, Birmingham, AL, USA \\ ${ }^{f}$ Center for Neurodegeneration and Experimental Therapeutics, University of Alabama at \\ Birmingham, Birmingham, AL, USA
}

\section{Abstract}

Background: Tools for efficient evaluation of amyloid-and tau-PET images are needed in both clinical and research settings.

Objective: This study was designed to validate a semi-automated image analysis methodology, called Biomarker Localization, Analysis, Visualization, Extraction, and Registration (BLAzER). We tested BLAzER using two different segmentation platforms, FreeSurfer (FS) and Neuroreader (NR), for regional brain PET quantification in participants in the Alzheimer's Disease Neuroimaging Initiative (ADNI) dataset.

Methods: 127 amyloid-PET and 55 tau-PET studies with volumetric MRIs were obtained from ADNI. The BLAzER methodology utilizes segmentation of MR images by FS or NR, then visualizes and quantifies regional brain PET data using FDA-cleared software (MIM), enabling quality control to ensure optimal registration and to detect segmentation errors.

\footnotetext{
${ }^{1}$ Data used in preparation of this article were obtained from the Alzheimer's Disease Neuroimaging Initiative (ADNI) database (http:// adni.loni.usc.edu). As such, the investigators within the ADNI contributed to the design and implementation of ADNI and/or provided data but did not participate in analysis or writing of this report. A complete listing of ADNI investigators can be found at: http:// adni.loni.usc.edu/wp-content/uploads/how to apply/AD NI Acknowledgement List.pdf

"Correspondence to: Jonathan McConathy, MD, PhD, Department of Radiology, University of Alabama at Birmingham, 619 19th St S, Birmingham, AL 35249, USA. Tel.: +1 205934 1388; jmcconathy@uabmc.edu.

Authors' disclosures available online (www.j-alz.com/manuscript-disclosures/19-0329r1).

SUPPLEMENTARY MATERIAL

The supplementary material is available in the electronic version of this article: http://dx.doi.org/10.3233/JAD-190329.
} 
Results: BLAzER analysis required $\sim 5$ min plus segmentation time. BLAzER using FS segmentation showed strong agreement with ADNI for global amyloid-PET standardized uptake value ratios (SUVRs) $(\mathrm{r}=0.9922, p<0.001)$ and regional tau-PET SUVRs across all Braak staging regions ( $\mathrm{r}>0.97, p<0.001$ ) with high inter-operator reproducibility (ICC >0.97) and nearly identical dichotomization as amyloid-positive or -negative (2 discrepant cases out of 127). Comparing FS versus NR segmentation with BLAzER, global SUVRs were strongly correlated for amyloid-PET ( $\mathrm{r}=0.9841, p<0.001)$, but were systematically higher ( $4 \%$ on average) with $\mathrm{NR}$, likely due to more inclusion of white matter with NR-defined regions.

Conclusions: BLAzER provides an efficient methodology for regional brain PET quantification. FDA-cleared components and visualization of registration reduce barriers between research and clinical applications.

\section{Keywords}

$\left[{ }^{18} \mathrm{~F}\right] \mathrm{AV}-45 ;\left[{ }^{18} \mathrm{~F}\right] \mathrm{AV}-1451$; amyloid- $\beta$; brain segmentation; neuroimaging; positron emission tomography; tau

\section{INTRODUCTION}

Positron emission tomography (PET) neuroimaging applications have increased in both research and clinical setting in recent years. PET provides the ability to study functional and molecular processes in the brain in vivo, allowing exploration of an array of normal and pathological states, including neurodegenerative disorders, psychiatric conditions, and neuro-oncology. Quantitation of PET data is well-established for research applications but less so in routine clinical settings. Recent research demonstrates the utility of quantification to supplement visual assessment for clinical PET, especially in Alzheimer's disease (AD) using the glucose analogue 2-deoxy-2-[ ${ }^{18}$ F]fluoro-D-glucose (FDG) and amyloid PET tracers [1-4]. Quantification of targeted brain regions of interest (ROIs) [5, 6] is enabled using 3-dimensional maps of regional brain anatomy generated by automated magnetic resonance imaging (MRI) segmentation algorithms, such as the widely used reference standard in research, FreeSurfer (FS) [7-9], or the FDA-cleared and ISO-certified Neuroreader (NR) [10-13]. By registering the segmentation map with the PET scan, ROIs can be defined for PET measurements without laborious and potentially imprecise or biased manual delineation methods [6, 14]. Barriers to the use of this approach to quantitation of brain PET, particularly for clinical applications, include computationally intensive software, time-consuming analysis procedures, the need for real-time visualization of the primary and processed data for clinical interpretation and quality control, and limited availability of FDA-cleared software suitable for this purpose.

Accuracy and precision are key features of effective image analysis methods to ensure that the extracted biomarker data can provide reliable in vivo information of the physiological state or disease process of interest. However, efficiency, ease of use, and availability are also essential features for widespread and routine implementation of quantitative brain PET. Many current techniques require lengthy post-processing time [2,15] and/or heavy computational workload [16, 17]. Incorrect segmentation of the brain MRI data and misregistration between PET and segmented brain regions are potential sources of error 
when extracting regional PET data based on ROIs defined through fully automated segmentation [15]. Additionally, although qualitative and quantitative assessment can be performed separately, the ability to easily visualize images in real-time at different states of processing and registration can provide an important degree of quality control and allow for user input to correct problematic studies. Many of the current fully automated processing methodologies that involve only inputs and outputs to optimize speed can be liable to unidentified errors because there is no opportunity to visualize the processed images [18, 19]. Finally, other barriers to routine implementation of quantitative brain PET include the lack of versatility in existing tools to analyze images acquired or processed by different platforms, radiotracers, and segmentation algorithms. Radiotracers commonly used for brain PET analysis in AD include $\left[{ }^{11} \mathrm{C}\right]$ Pittsburgh compound $\mathrm{B}(\mathrm{PiB})[20],\left[{ }^{18} \mathrm{~F}\right]$ florbetapir [21, 22], $\left[{ }^{18} \mathrm{~F}\right]$ florbetaben [23], and $\left[{ }^{18} \mathrm{~F}\right]$ flutemetamol [24] for amyloid-p (Ap), $\left[{ }^{18} \mathrm{~F}\right]$ flortaucipir for tau [25-28], and $\left[{ }^{18} \mathrm{~F}\right] \mathrm{FDG}[29,30]$ for glucose metabolism. Multicenter clinical trials and longitudinal studies can benefit from a versatile methodology to standardize PET quantification [31].

Although a number of effective research methodologies exist for regional brain PET analysis that combine one or more of the previously discussed features [8, 19, 32-35], quantification still has not been widely implemented in routine clinical brain PET for AD, which relies primarily on visual assessment and components validated for routine clinical use. FDA clearance and ISO-certification are key features of NR, a commercial service that provides automated brain MRI segmentation for clinical use, and MIM, a commercial software package for image visualization and quantification. Combining features of such tools could help lower the barriers between clinical and research applications. However, to the best of our knowledge, no method exists that is efficient and easy for physicians and technologists to use, automatically segments ROIs in a customizable fashion, consists of FDA-cleared components, and allows for both qualitative and quantitative assessment of brain PET data in a clinical environment.

We present here a novel brain Biomarker Localization, Analysis, Extraction, and Registration (BLAzER) methodology for analysis of PET based on segmented brain MRI. We demonstrate that BLAzER works well for both global and regional PET quantification of brain amyloid-and tau-PET, respectively, in Alzheimer's Disease Neuroimaging Initiative (ADNI) participants spanning normal cognition, mild cognitive impairment (MCI), and AD dementia. Although this report focuses on AD biomarkers, BLAzER could be applied to any PET neuroimaging study that utilizes MR-based segmentation for defining ROIs.

Additionally, we compare two different inputs for segmentation, FS and NR, which could be used with BLAzER for research and clinical applications, respectively.

\section{METHODS}

\section{Study population}

Images were downloaded from the Alzheimer's Disease Neuroimaging Initiative (ADNI) database (http://www.loni.ucla.edu/ADNI/). ADNI was launched in 2003 as a public-private partnership, led by principal investigator Michael W. Weiner, MD. The primary goal of ADNI has been to test whether serial magnetic resonance imaging (MRI), PET, other 
biological markers, and clinical and neuropsychological assessment can be combined to measure the progression of $\mathrm{MCI}$ and early $\mathrm{AD}$. All studies were approved by the local Institutional Review Board at each institution through ADNI. Additional information is available at the ADNI website (http://www.adni-info.org).

We chose to use the quantitative amyloid-and tau-PET analyses available through ADNI previously performed by the University of California Berkeley (UC Berkeley) group as the reference standard for BLAzER using FreeSurfer for segmentation. At the time of our analysis, this data set consisted primarily of florbetapir (also known as AV-45) scans for amyloid-PET and flortaucipir (also known as AV-1451) scans for tau-PET. A total of 127 amyloid-PET and 55 tau-PET studies were selected from ADNI for a total of 178 unique subjects (4 overlap). Both sets of subjects were selected to represent the spectrum of ADNI participants in terms of cognitive status (cognitively normal (CN), MCI, and AD), age, and brain amyloid and tau pathology. Our age ranges for amyloid-PET studies (cohorts aged 55$59,60-69,70-79$, and 280 ) and tau-PET studies (cohorts aged 60-69, 70-79, and $\geq 80$ ) differed slightly due to the population available in ADNI at time of analysis. The demographics of subjects included in BLAzER analysis are presented in Table 1.

In the case of the amyloid-PET cohort, subjects meeting the age and cognitive status requirements were selected for inclusion in order of ascending subject ID in ADNI. In the case of the tau-PET cohort, ADNI did not contain enough studies at the time of analysis to match the same age groups as for the amyloid-PET cohort, and subjects were selected randomly within each cognitive status classification. Selection for all subjects was blinded to the quality of the PET and MRI scans as well as the quantitative analysis available in ADNI. Image analysis with BLAzER was performed blind to subjects' cognitive status and age group. No subjects were excluded at the time of selection or subsequently during the analysis; all of the initially selected studies were included in the final analysis.

\section{Image sets used for analysis}

All subjects in both the amyloid-PET and tau-PET cohorts had a volumetric brain MRI scan performed within 1 year of the PET study. MRI studies were performed using standard T1weighted sagittal 3D MPRAGE (magnetization prepared rapid gradient echo) sequences acquired from various 3T scanners with a $1.25 \times 1.25-\mathrm{mm}$ in-plane spatial resolution and 1.2 -mm slice thickness with $256 \times 256$ voxel resolution according to ADNI specifications.

Amyloid-and tau-PET studies were acquired with $\left[{ }^{18} \mathrm{~F}\right]$ florbetapir and $\left[{ }^{18} \mathrm{~F}\right]$ flortaucipir, respectively. PET images included in ADNI were obtained on different scanners of varying resolutions, each with its platform-specific acquisition protocol. Therefore, as part of the ADNI protocol, all raw PET images undergo pre-processing for quality control and standardization purposes at the University of Michigan [36]. In summary, $4 \times 5$ min dynamic image frames, acquired 50 to $70 \mathrm{~min}$ post-injection, were co-registered to the first extracted frame of the raw image file. Then, the 6 five-min frames were averaged to form a static PET image and reoriented into a standard $160 \times 160 \times 96$ voxel image grid with $1.5-\mathrm{mm}$ cubic voxels. Finally, each image set was filtered with a scanner-specific filter function to produce images of uniform isotropic resolution of $8 \mathrm{~mm} \mathrm{FWHM,} \mathrm{which} \mathrm{is} \mathrm{the} \mathrm{approximate}$ resolution of the lowest resolution scanners used in ADNI. Only the fully pre-processed, 
standardized, co-registered, and averaged PET images were used for this study. Further details on the ADNI acquisition protocol are available on the website (http:// adni.loni.usc.edu/methods).

\section{Image analysis}

The key components needed to implement the BLAzER methodology are summarized in the Supplementary Materials, and the custom-built MIM workflows used for the BLAzER analyses in this work are available for download.

\section{MRI segmentation}

Volumetric MRI images were segmented by either FreeSurfer v6.0.0 (Boston, MA) or Neuroreader (Brainreader, Horsens, Denmark). FS, an open-source software, has been validated as a tool to measure brain volumes in various neurological diseases when compared to either manual delineation [6] or other algorithms [14]. FS uses a complex algorithm with a series of normalization and motion-correction approaches prior to even starting its intensity-based approach to parcellate the brain into not only the larger cortical structures, but also subregions to allow targeted measurement of specific ROIs [8,9]. FS segmentation produces detailed ROIs, which allow for spatial delineation of brain regions beyond the main lobar cortical and subcortical brain structures. Customization of brain regions is particularly useful in analysis of tau-PET studies in Alzheimer's disease where pathology follows characteristic Braak staging regions [37].

NR's separation of gray matter, white matter, and cerebrospinal fluid uses an intensity-based approach along with a template, similar to other available approaches [38,39] such as the widely used Automatic Anatomic Labeling (AAL) template [19]. Similar to AAL, NR is highly efficient, with segmentation of an individual case processed in approximately $15 \mathrm{~min}$ compared to 8 to $12 \mathrm{~h}$ for FS. However, segmented brains from NR provide larger cortical and subcortical brain regions compared to FS, limiting customization beyond anatomical boundaries defined by their atlas. NR's atlas differs from FS's Deskian/Killiany atlas but is held as proprietary information by Neuroreader.

FS studies were loaded onto a supercomputer environment (Cheaha, Birmingham, AL) in order to run all 184 volumetric MRI input files simultaneously through parallel computing. For efficient parallel processing viaFreeSurfer, DICOM images were converted to NIfTI using MRIcron, Release 2May2016 (https://www.nitrc.org/projects/mricron). Upon completion of segmentation, parcellated, segmented brain images were converted from MGZ to DICOM format using 3DSlicer v4.5.0 (Boston, MA). Alternatively, NR automatically produced segmented brains in DICOM format for the same MPRAGE scans without requiring conversion between file formats. Each of the segmentation output files along with source volumetric MRI and pre-processed PET scans were then visualized and quantified using a customized work-flow in the commercial, multi-modal FDA-cleared imaging software - MIM v6.6.13 (MIM Software Inc., Cleveland, OH). Through a graphical user interface (GUI), MIM allows the user to design customized, automated algorithms (called workflows) for processing primary imaging data without the need for advanced computational skills. 


\section{PET analysis}

We developed workflows that could use segmentation data from FS or NR as inputs for ROI definition on the PET data sets. For BLAzER, we selected the segmented brain dataset, brain PET, and volumetric brain MRI images for analysis, then ran a user-defined and customizable workflow in MIM that registered the images, defined ROIs within the original volumetric MR and PET using the segmented brain dataset, and delivered the quantification in an array of customizable outputs while allowing for visual inspection during the workflow for quality control. The workflows in MIM were developed and customized to the specific segmentation methods (FS and NR) and PET radiotracers through a GUI interface where steps in the protocol were simply selected from a menu similar to Porcupine [40], such that users were not limited by their computational skillset to analyze large, multi-modal image datasets.

A key aspect of the MIM workflows is assigning ROIs to specific brain regions based on the segmented brain dataset that can be applied to the PET and volumetric MR images for quality control and quantitative analysis as shown schematically in Fig. 1. In both FS and $\mathrm{NR}$, each brain region represented in the segmentation dataset is assigned a unique pixel value that is atlas-specific. For example in FS, all pixels that constitute the left precuneus are assigned the arbitrary value 1025 . The workflow defines each ROI based on these atlasspecific values and then applies each ROI to the co-registered PET and volumetric MRI data sets. In some cases, multiple segmented brain ROIs are combined to define a larger brain ROI (e.g., lobar cortical regions, Braak staging regions). The pixel values defining each ROI as well as how the smaller ROIs are combined into larger ROIs are readily customizable in the workflows to match atlas-specific segmentation definitions and the intended application.

In summary, in the BLAzER methodology, we 1) segmented the MRI to generate a 3D brain segmentation map using either FS or NR; 2) transferred the segmentation to MIM and selected corresponding volumetric MRI and PET for analysis; 3) automatically co-registered the brain segmentation map to volumetric MRI; 4) utilized the segmentation map to delineate the various brain ROIs based on the pixel intensities for each region as defined by FS or NR; 5) performed quality control through visualization and corrected registration, if necessary; and 6) fused PET scans to MRI/brain segmentation maps to extract, visualize, and quantify PET data from the workflow-defined ROIs (Fig. 1).

The methodology suspended the automated process before transferring the contours to allow the user to verify accurate image registration and identify any segmentation errors. The original volumetric MRI with the segmentation-based ROIs superimposed were used for quality control measure to assure that the rigid registration method used to align the images had been performed properly and the brain segmentation were correct. If needed, the user could manually correct the registration and/or ROIs at this step. We did not manually change any ROIs for any studies in the data analysis presented in this manuscript even when minor segmentation errors were apparent. This review step was employed for all cases but is optional and could be omitted to provide a more automated process. Inter-operator variability for BLAzER was assessed by having two individuals independently process the same randomly selected subsets of 40 amyloid-PET, 30 tau-PET, and 40 volumetric MRI studies and then comparing the quantitative results between the two operators. 


\section{Anatomical definitions of brain regions}

In BLAzER, all SUVRs extracted are automatically weighted by the volumes of the individual subregions comprising each ROI. Amyloid-PET ROIs were normalized to the entire cerebellum to extract SUVRs for all regions that comprised the cerebral cortex: frontal lobe, temporal lobe, parietal lobe, and cingulate (BLAzER-FS, as defined by ADNI) or frontal, temporal, parietal, and occipital lobes (BLAzER-NR, as defined by Neuroreader). BLAzER-FS matched UC Berkeley's ADNI-defined cortical regions exactly while BLAzER-NR included the entire cortex. For head-to-head comparisons between BLAzER and ADNI, BLAzER-FS delineation was based on UC Berkeley's ADNI-defined brain subregions. However, for comparisons between BLAzER-FS and BLAzER-NR, BLAzERFS anatomical regions were modified to match the NR-based regions of entire cortex. We denote this distinction as BLAzER-FS*. Specific FreeSurfer subregions are detailed below as well as listed in Supplementary Table 1.

Specifically, BLAzER-FS subregions were defined as follows: caudal middle frontal, lateral orbitofrontal, medial orbitofrontal, pars opercularis, pars orbitalis, pars triangularis, rostral middle frontal, superior frontal, and frontal pole (frontal); caudal anterior cingulate, isthmus cingulate, posterior cingulate, and rostral anterior cingulate (cingulate); inferior parietal, precuneus, superior parietal, and supramarginal (parietal); middle temporal and superior temporal (temporal). In contrast, BLAzER-FS* regions were defined as follows: caudal middle frontal, lateral orbitofrontal, medial orbitofrontal, pars opercularis, pars orbitalis, pars triangularis, rostral middle frontal, superior frontal, frontal pole, paracentral gyrus, precentral gyrus, caudal anterior cingulate, posterior cingulate, and rostral anterior cingulate, and insula (frontal); inferior parietal, pre-cuneus, superior parietal, supramarginal, postcentral gyrus, and isthmus cingulate (parietal); bankssts, entorhinal, fusiform, inferior temporal, middle temporal, parahippocampal, superior temporal, temporal pole, and transverse temporal (temporal); cuneus, lateral occipital, lingual, and pericalcarine (occipital).

Tau-PET SUVRs were calculated similarly to the amyloid-PET defined ROIs with two main differences. Tau-PET was normalized to the cerebellar gray matter instead of the entire cerebellum [37] and UC Berkeley's ADNI-defined ROIs for BLAzER-FS methodology followed the pathologic Braak staging regions [41]: entorhinal cortex (Braak 1); hippocampus (Braak 2); parahippocampal, fusiform, lingual, and amygdala (Braak 3); middle temporal, caudal anterior cingulate, rostral anterior cingulate, posterior cingulate, isthmus cingulate, insula, inferior temporal, and temporal pole (Braak 4); superior frontal, lateral orbitofrontal, medial orbitofrontal, frontal pole, caudal middle frontal, rostral middle frontal, pars opercularis, pars orbitalis, pars triangularis, caudate, putamen, lateral occipital, parietal supramarginal, inferior parietal, superior temporal, pallidum, superior parietal, precuneus, superior temporal sulcus, nucleus accumbens, and transverse temporal (Braak 5); pericalcarine, postcentral, cuneus, precentral, and paracentral (Braak 6). As NR's anatomical atlas only includes major cortical and subcortical regions, we defined BLAzER-NR and BLAzER-FS* tau-PET regions the same as for amyloid-PET: frontal, temporal, parietal, and occipital cortices. 
The previously processed ADNI data from the UC Berkeley group was used as the reference standard. The reported values in the ADNI data set were based on brain segmentations performed using FS v5.3.0. SUV and SUVR data was extracted using SPM5 after coregistration of PET and MR data. Key differences between BLAzER and the ADNI method are summarized in Table 2.

\section{Statistical analysis}

Statistical analyses were performed using Med-Calc v17.7.2 (MedCalc Software, Mariakerke, Belgium) and Matlab vR2016b (MathWorks, Natick, MA) to compare the BLAzER method with the reference standard from ADNI. SUVRs and volumes were reported as mean $\pm \mathrm{SD}$. Statistical significance was defined as $p<0.002$ to account for up to 25 multiple comparisons using a Bon-ferroni correction. Univariate regression was used to validate measurements between the two methods using Pearson's correlation coefficient (poor agreement $=0 ;$ slight $=0.01-0.20 ;$ fair $=0.21-0.40 ;$ moderate $=0.41-0.60 ;$ good $=$ $0.61-0.80$, and excellent $=0.81-1.00$ agreement). Similar comparisons were conducted using Bland-Altman evaluations to determine limits of agreement and percent differences between the two analysis tools. Intra-and inter-observer variability and agreement were evaluated for global cortical SUVR using the intraclass correlation coefficient (ICC) in a two-way random model (ICC $<0.40=$ poor; ICC $\geq 0.40$ to $0.75=$ fair to good; ICC $>0.75=$ excellent agreement). Finally, amyloid-PET cases were dichotomized into positive or negative to determine the ability of BLAzER to properly classify cases based on SUVR cutoffs. Dichotomization was performed by 1) directly applying ADNI's autopsy-derived 1.11 cutoff $[34,42]$ and 2) deriving BLAzER-specific cutoff by using a linear regression to convert "ADNI units" to BLAzER units based on the slope and y-intercept.

\section{RESULTS}

\section{Efficiency, quality control, and reproducibility}

The BLAzER methodology enabled rapid image analysis. The most time-consuming step was segmentation. NR was considerably faster than FS on a per-case basis (10-20 min/case versus 8-12 h/case, respectively), yet slower than FS for total processing time for the 182 subject cohort ( $45.5 \mathrm{~h}$ versus $12 \mathrm{~h}$, respectively) due to the ability to run FS cases in parallel on a supercomputer environment. After segmentation, FS cases required $\sim 2$ min for manual DICOM conversion whereas NR cases were returned in DICOM format. Once the segementation was obtained, the remainder of BLAzER processing took about 5 min per case. Thus, a case could be fully processed, from completion of image acquisition to full regional quantification, in as little as 20 min when using NR segmentation.

Although the methodology could operate with full automation, the ability to visualize registration provided for quality control and avoiding registration errors. We routinely ran MIM's "Run Rigid Assisted Alignment" tool serially to fix minor errors until it provided no further adjustment, which took only a few seconds (Fig. 1). Only 1 of the 182 scans required manual correction of registration, which was a case with severe brain atrophy. This example highlights the usefulness of the visualization step as the incorrect registration would have been missed in a fully automated methodology. We also examined inter-operator reliability 
by having two independent operators process each scan. Global florbetapir-and regional flortaucipir-PET measurements showed excellent reproducibility between two users across all brain regions (ICC $>0.97$, Table 3).

\section{MRI volumetric measurements}

As BLAzER's regional PET extraction depends on accurate MRI-based anatomic segmentation and registration, we first compared BLAzER measurements of regional MRI volumes to the ADNI reference standard. Global cortical volume measured by the BLAzER methodology was highly correlated to that measured by ADNI $(r=0.9749, p<0.001$, Fig. $2 \mathrm{~A}$ ) with small systematic difference $(1.61 \%)$ and tight $95 \%$ confidence interval (CI) (Fig. 2B). Regional comparisons across the frontal, cingulate, parietal, and temporal lobes for $\mathrm{CN}$, MCI, and AD subjects showed similar results $(r>0.92, p<0.0001$, Supplementary Table 2).

\section{Amyloid-PET SUVR and dichotomization}

BLAzER showed strong agreement with the ADNI reference standard when measuring diffuse cortical binding of amyloid-PET. Global SUVRs were similar between BLAzER and ADNI ( $r=0.9922, p<0.0001$, Fig. 3A). Additionally, the slope of linear regression and $y-$ intercept $\left(\mathrm{y}=1.0012 \mathrm{x}+0.01816, \mathrm{R}^{2}=0.9844\right.$, Table 4) showed a near one-to-one correspondence between BLAzER and ADNI. There was a slight systematic difference in global SUVR, which was on average 1.6\% higher with BLAzER than in the ADNI dataset (Fig. 3B). This may be due to a systematic reduction in the reference region mean, which was observed in the cerebellum. Regional comparisons across the frontal, cingulate, parietal, and temporal lobes for $\mathrm{CN}, \mathrm{MCI}$, and $\mathrm{AD}$ subjects showed similar results $(\mathrm{r}>0.94, p<$ 0.0001, Supplementary Table 3).

We also the examined the dichotomous classification of individuals as amyloid-positive versus negative based on SUVR cutoffs. Because BLAzER SUVRs were slightly higher than ADNI on average, a few more of the 127 subjects were classified as amyloid-positive when BLAzER SUVRs were dichotomized using the ADNI cutoff of 1.11 with all 4 of these cases lying near the cutoff (BLAzER versus ADNI range: [1.113 to 1.125] versus [1.060 to 1.098], respectively, Table 4). However, translating 1.11 "ADNI units" into 1.13 "BLAzER units" by linear regression (Equations 1 and 2) provided the same dichotomization as ADNI for these 4 cases, but 2 different cases were discrepant out of the 127 total at this cutoff. These 2 cases had SURVs of 1.113 versus 1.125 and 1.113 versus 1.115 for BLAzER-FS versus ADNI, respectively.

$$
\begin{gathered}
S U V R=\frac{S U V_{\text {target region }}}{S U V_{\text {whole cerebellum }}} 1 . \\
S U V R_{B L A z E R}=S U V R_{A D N I} * \text { slope }_{B L A z E R}+\text { intercept }_{B L A z E R} 2 .
\end{gathered}
$$




\section{Regional brain tau-PET comparison}

BLAzER showed strong agreement with the ADNI reference standard when looking at regional binding of tau-PET with flortaucipir. Despite slightly lower correlation coefficients and wider 95\% confidence intervals than with amyloid-PET (as expected because of the smaller regions defined for analysis), BLAzER correlated strongly with the ADNI reference standard across all six Braak stage regions ( $r>0.97, p<0.0001$, Fig. 4). Further demonstrating regional accuracy, BLAzER showed tight $95 \% \mathrm{CI}(<10 \%)$ and small differences (-1.5 to 3.6\%) across all regions (Fig. 5), and all cognitive statuses ( $\mathrm{r}>0.91, p$ $<0.0001$, Supplementary Table 3).

\section{Amyloid and tau PET quantitation based on NR segmentation}

Next we compared the performance of a different segmentation platform, comparing BLAzER-FS with BLAzER-NR. To enable a more direct comparison, we first adjusted FS's anatomical subregions to match NR's composite regions (FS*, see Methods). BLAzER-NR highly correlated with BLAzER-FS* at a global level for amyloid-PET $(\mathrm{r}=0.9841, p<$ 0.001 , Fig. 6A). However, BLAzER-NR values had higher SUVR than BLAzER-FS* as shown by the systematic difference (4.0\%) and shifted $95 \%$ CI (-0.7\% to $8.7 \%$, Fig. 6B). This is likely due to BLAzER-NR segmentation including more PET signal from white matter, which has high florbetapir non-specific binding, due to a slightly thicker definition of cortical regions with NR compared to FS. Dichotomization results paralleled these findings (Table 5). Directly applying ADNI's cutoff of 1.11 led to 12 out of 127 cases being discrepant between BLAzER-NR and BLAzER-FS* (range of SUVRs: [1.092 to 1.180] versus [1.049 to 1.111], respectively). After applying regression analysis for both BLAzERNR to ADNI $\left(\mathrm{y}=0.9845 \mathrm{x}+0.0 .05658, \mathrm{R}^{2}=0.9587\right)$ and BLAzER-FS to ADNI $(\mathrm{y}=$ $0.8947 \mathrm{x}+0.1158, \mathrm{R}^{2}=0.9482$ ), the new SUVR cutoff obtained was 1.15 for BLAzER-NR while BLAzER-FS* remained unchanged. The adjusted cutoff reduced the number of discrepant cases to 7 out of 127 (NR versus FS range: [1.0923 to 1.180] versus [1.088 to 1.120], respectively). This discrepancy reflects the differences between the segmentation provided by NR and FS which in turn affects SUVRs.

Regional analysis for both amyloid-and tau-PET across different cognitive statuses and brain regions revealed excellent correlations between BLAzER-NR and BLAzER-FS* $(r>0.85, p$ $<0.0001$, Supplementary Table 4) although not as high as the correlations between BLAzER-FS and the ADNI reference values, again reflecting differences in the segmentation between the NR and FS platforms.

\section{DISCUSSION}

Measurement of the regional brain distribution of PET tracers is a cornerstone of data analysis in molecular neuroimaging and is growing in importance for clinical applications, particularly in the evaluation of patients with cognitive impairment. A number of imaging processing methodologies exist that perform quantification of regional brain PET data $[8$, 19, 32-35]. However, there are few if any analysis tools that perform well for both clinical and research applications, utilize FDA-cleared components, define ROIs and extract regional PET data automatically, provide both quantitative and real-time visual assessment, and work 
quickly and efficiently without need for advanced coding skills. Our evaluation of BLAzER with validation through comparison to the ADNI database and head-to-head comparison of two segmentation methods demonstrates an approach that addresses this unmet need.

Quantitative brain PET data from BLAzER correlated very closely with the results in ADNI for florbetapir-and flortaucipir-PET analyzed using FreeSurfer-defined regional volumes.

We also demonstrated that the regional brain volumes provided by FS and NR were in close agreement with ROIs defined through BLAzER with less than $2 \%$ error between the original volumes and those output by BLAzER. BLAzER and ADNI produced nearly identical dichotomous classification of amyloid-PET participants as positive or negative, especially when using regression analysis to account for systematic differences. It is important to acknowledge that our analysis was completed before the most recent ADNI update, which includes a new partial volume corrected set of values based on modified reference regions. Thus, any partial volume effect (PVE), or inaccuracies that result from low PET spatial resolution [43], affected both BLAzER and our reference ADNI set similarly. In addition to PVE, off-target-binding in non-specific regions can be problematic for accurate PET quantification. Recent evidence has shown non-specific flortaucipir uptake in the basal ganglia $[44,45]$. In the most recent ADNI updates, the caudate and putamen have been removed from the Braak 5 staging regions for flortaucipir. However, we kept our results consistent by comparing BLAzER to the original dataset that included the caudate and putamen. In future work, BLAzER-FS regions can be modified to account for PVE and offtarget-binding if desired.

We observed slightly larger systematic differences and CIs for flortaucipir-PET compared to florbetapir-PET. As pathological cortical tau distribution is more regionally localized and restricted than pathological cortical amyloid which tends to be diffuse throughout the cerebral cortex [28], differences in segmentation and image alignment are expected to have a greater effect on PET measures of regional brain tau compared to regional brain amyloid.

Potential sources of the small differences between BLAzER and the ADNI data set include different FS versions, source volumetric MR images, and registration methods. For our study, FS v6.0.0 was used while v5.3.0 was used for the ADNI data set, which could affect anatomical volume measurements and brain segmentation which in turn would affect ROI definition for PET quantitation [46]. Additionally, the ADNI dataset used two volumetric MR scan input files for FS to produce the segmented brain while we chose to use only one volumetric brain MR to reflect our standard research and clinical protocols. Within ADNI, each subject undergoes two volumetric MR scans during a single scanning session with some subject datasets having slightly different imaging parameters. In order to align the PET and MR images, ADNI utilized SPM to register the images automatically while we used MIM. BLAzER utilizes a rigid-registration algorithm that allows only translation and rotation. Additionally, we utilize a three-step system of registration where the segmentation is first registered with its original, template volumetric MR and then with the PET after the rigid-registration quality control-check. We believe that this visual assessment and option for manual correction minimizes sources of error that can arise from fully automated registration methods (Fig. 1) [2]. Additionally, we believe that our results are generaliz-able 
across the ADNI population due to our selection of individuals that represent the range of amyloid and tau pathology in ADNI.

Our implementation of BLAzER for research studies utilizes a high-throughput, supercomputing cluster for segmentation (by FS) along with a commercially available multimodality image viewing and analysis software program (MIM) with customized methodologies to use and process the segmentation data. This methodology can be adapted to other segmentation algorithms by customizing the visualization software workflow with minimal change in the user experience. This methodology allows the user to register and process brain segmentation maps with brain PET and brain MRI in a single viewing environment for quality control, manual correction if needed, data extraction, and real-time visual assessment. Utilizing parallel computing strategies with FS allows segmentation of over 100 studies in approximately $12 \mathrm{~h}$ which is suitable for use with clinical trials and large imaging datasets. We chose MIM due to its efficiency and relative ease of use compared to existing tools that are either dependent on relatively heavy computational skills [16, 17] or require significant image processing input [2,15]. MIM's GUI-interface is most similar to Porcupine [40], enabling analysis of large, multi-modal image datasets by users without a sophisticated computational skillset. Similar to other existing methodologies [2, 47-49], BLAzER allows for automatic delineation of PET ROIs. However, an important advantage of BLAzER is that it can accept brain segmentation data sets from multiple sources, including FDA-cleared algorithms such as NR. This potential integration of completely FDA-cleared components when utilizing BLAzER helps overcome barriers between clinical and research applications of BLAzER. This method can accept any brain segmentation map in DICOM format as in the case of NR or segmentation maps that can be converted to DICOM format as in the case of FS.

While the present work focuses on the use of this methodology for amyloid-and tau-PET, it is applicable for the analysis of other brain PET imaging studies. This flexibility combined with visualization software familiar to physicians and technologists facilitates implementation of quantitative PET neuroimaging in routine clinical practice with a wide range of segmentation algorithms and PET tracers. As BLAzER can accommodate any DICOM dataset, other neuroimaging modalities, such as ioflupane-SPECT for Parkinson's disease or functional MRI and diffusion tensor imaging for schizophrenia, could be processed along with PET neuroimaging biomarkers of neuroinflammation [50-52] by adapting the BLAzER methodology. Although not performed in this study, BLAzER could be used for analysis of dynamic PET data sets as well as centiloid analysis of amyloid-PET data. The centiloid process uses a 0 to 100 unit scale with healthy controls ( $<45$ years of age) to set the 0 unit anchor and amyloid-positive studies from individuals with typical AD to set the 100 unit anchor. A key advantage of centiloid scales is the ability to combine data from different PET scanners, amyloid-PET tracers, and analysis methods. Although not performed in the current study, the BLAZeR parameters including brain regions of interest and SUVR scaling could be adapted to match the centiloids methodology described by Klunk et. al. to provide results on a centiloid scale [53].

Another major strength of the BLAzER methodology is the ability to use any brain segmentation map in DICOM format for defining PET ROIs and extracting PET 
measurements. However, in contrast to our inter-methodology comparison between BLAzER-FS and ADNI, our intra-methodology comparison between BLAzER-FS* and BLAzER-NR demonstrated subtle differences between the segmentation methods, yet still showed excellent correlations for both global and regional comparisons. Our results showed that NR provides slightly higher SUVRs when compared to FS across large cortical structures, where dichotomous classification revealed 7 discrepant individuals out of 127 total cases even after regression adjustment. These findings, which contrast with the nearly identical dichotomization between BLAzER-FS and ADNI comparison, emphasize the effect that differences in segmentation can have on amyloid dichotomization. Of note, the discrepant dichotomous classifications of amyloid status using both FS and NR segmentation involved cases with SUVRs very close to the cut-off value. Despite our best efforts to match BLAzER-NR's cortical anatomical boundaries with rearrangement of BLAzER-FS* regions, BLAzER-NR and BLAzER-FS* have slightly different anatomical definitions of brain subregions across cortical structures not only from each other but also from ADNI (unlike BLAzER-FS), which noticeably affects correlation and dichotomization. Additionally, the methods utilize different segmentation algorithms in separating gray from white matter, leading to slightly thicker cortical ROIs with NR than with FS. As florbetapir binds non-specifically to white matter even in cognitively normal individuals [54], we believe that a greater contribution of PET signal from white matter in BLAzER-NR contributes to the systematically larger SUVRs when compared to BLAzER-FS*. Based on our observed results, the selection of the appropriate segmentation input depends on the application. For rapid analysis of large major cortical or subcortical structures in a clinical setting, NR and similar algorithms might be preferred. In contrast, FS provides more versatility for brain segmentation due its ability to provide smaller ROIs compromised of multiple sub-regions which may be more appropriate for research and analysis of PET tracers with spatially-restricted distributions.

There are limitations to the current implementation of BLAzER. First, the only file format accepted by the commercial software MIM is DICOM, while other file formats (e.g., NIfTI) are often used in the research community when working with large imaging data sets. Thus, file format conversion is required if the output of a brain segmentation methodology is not in DICOM format as in the case of FS. Second, there are manual components to the quality control that require user review and confirmation to proceed. Many of these user inputs could be removed as intervention is only needed when an error is identified, but the quality of the resulting PET quantification and the identification of segmentation errors would likely suffer. Finally, the use of commercial software in the BLAzER methodology increases the cost when compared to some other software options for brain PET analysis. However, time savings in terms of user training and study processing should be factored into the comparison between costs of different methodologies. Additionally, the use of FDA-cleared software, often more expensive than software used for research only, is important when considering implementing this methodology for quantification of PET data into routine clinical studies.

In conclusion, BLAzER is a streamlined image processing methodology for efficient registration, visualization, and extraction of brain PET data. We successfully validated the accuracy and reproducibility of BLAzER using ADNI as the reference standard for amyloid- 
PET and tau-PET analyses. We also showed how two different segmentation inputs, NR and FS, can be used with BLAzER and that the cortical ROIs extracted by BLAzER remain consistent with the original MR volumes. This versatile methodology can reduce barriers to quantitative brain PET and MR analysis for a wide range of research and clinical applications.

\section{Supplementary Material}

Refer to Web version on PubMed Central for supplementary material.

\section{ACKNOWLEDGMENTS}

Data used in the preparation of this article were obtained from the ADNI database (http://adni.loni.usc.edu), which is easily available for download from the Laboratory of Neuroimaging (LONI) website to the research public.

This work was supported by National Institutes of Health grants RF1AG059009 and T32GM008361, the Alzheimer's Drug Discovery Foundation, and the Department of Radiology at the University of Alabama at Birmingham. Data collection and sharing for this project was funded by the Alzheimer's Disease Neuroimaging Initiative (ADNI) (NIH U01AG024904) and DOD ADNI (Department of Defense award number W81XWH-12-2-0012). ADNI is funded by the National Institute on Aging, the National Institute of Biomedical Imaging and Bioengineering, and through generous contributions from the following: AbbVie, Alzheimer's Association; Alzheimer's Drug Discovery Foundation; Araclon Biotech; BioClinica, Inc.; Biogen; Bristol-Myers Squibb Company; CereSpir, Inc.; Cogstate; Eisai Inc.; Elan Pharmaceuticals, Inc.; Eli Lilly and Company; EuroImmun; F. Hoffmann-La Roche Ltd and its affiliated company Genentech, Inc.; Fujirebio; GE Healthcare; IXICO Ltd.; Janssen Alzheimer Immunotherapy Research \& Development, LLC.; Johnson \& Johnson Pharmaceutical Research \& Development LLC.; Lumosity; Lundbeck; Merck \& Co., Inc.; Meso Scale Diagnostics, LLC.; NeuroRx Research; Neurotrack Technologies; Novartis Pharmaceuticals Corporation; Pfizer Inc.; Piramal Imaging; Servier; Takeda Pharmaceutical Company; and Transition Therapeutics. The Canadian Institutes of Health Research is providing funds to support ADNI clinical sites in Canada. Private sector contributions are facilitated by the Foundation for the National Institutes of Health (http://www.fnih.org). The grantee organization is the Northern California Institute for Research and Education, and the study is coordinated by the Alzheimer's Therapeutic Research Institute at the University of Southern California. ADNI data are disseminated by the Laboratory for Neuro Imaging at the University of Southern California.

\section{REFERENCES}

[1]. Jack CR Jr, Wiste HJ, Weigand SD, Therneau TM, Lowe VJ, Knopman DS, Gunter JL, Senjem ML, Jones DT, Kantarci K, Machulda MM, Mielke MM, Roberts RO, Vemuri P, Reyes DA, Petersen RC (2017) Defining imaging biomarker cut points for brain aging and Alzheimer's disease. Alzheimers Dement 13, 205-216. [PubMed: 27697430]

[2]. Hutton C, Declerck J, Mintun MA, Pontecorvo MJ, Devous MD Sr., Joshi AD, Alzheimer's Disease Neuroimaging Initiative (2015) Quantification of ${ }^{18}$ F-florbetapir PET: Comparison of two analysis methods. Eur J Nucl Med Mol Imaging 42, 725-732. [PubMed: 25652817]

[3]. Bullich S, Seibyl J, Catafau AM, Jovalekic A, Koglin N, Barthel H, Sabri O, De Santi S (2017) Optimized classification of ${ }^{18}$ F-Florbetaben PET scans as positive and negative using an SUVR quantitative approach and comparison to visual assessment. Neuroimage Clin 15, 325-332. [PubMed: 28560157]

[4]. Minoshima S, Koeppe RA, Frey KA, Ishihara M, Kuhl DE (1994) Stereotactic PET atlas of the human brain: Aid for visual interpretation of functional brain images. JNucl Med 35, 949-954. [PubMed: 8195881]

[5]. Destrieux C, Fischl B, Dale A, Halgren E (2010) Automatic parcellation of human cortical gyri and sulci using standard anatomical nomenclature. Neuroimage 53, 1-15. [PubMed: 20547229]

[6]. Lehmann M, Douiri A, Kim LG, Modat M, Chan D, Ourselin S, Barnes J, Fox NC (2010) Atrophy patterns in Alzheimer's disease and semantic dementia: A comparison of FreeSurfer and manual volumetric measurements. Neuroimage 49, 2264-2274. [PubMed: 19874902]

[7]. Fischl B (2012) FreeSurfer. Neuroimage 62, 774-781. [PubMed: 22248573] 
[8]. Fischl B, Salat DH, Busa E, Albert M, Dieterich M, Haselgrove C, van der Kouwe A, Killiany R, Kennedy D, Klaveness S, Montillo A, Makris N, Rosen B, Dale AM (2002) Whole brain segmentation: Automated labeling of neuroanatomical structures in the human brain. Neuron 33, 341-355. [PubMed: 11832223]

[9]. Fischl B, Salat DH, van der Kouwe AJ, Makris N, Segonne F, Quinn BT, Dale AM (2004) Sequence-independent segmentation of magnetic resonance images. Neuroimage 23(Suppl 1), S69-84. [PubMed: 15501102]

[10]. Ahdidan J, Raji CA, DeYoe EA, Mathis J, Noe KO, Rimestad J, Kjeldsen TK, Mosegaard J, Becker JT, Lopez O (2016) Quantitative neuroimaging software for clinical assessment of hippocampal volumes on MR imaging. J Alzheimers Dis 49, 723-732. [PubMed: 26484924]

[11]. Tanpitukpongse TP, Mazurowski MA, Ikhena J, Petrella JR, Alzheimer's Disease Neuroimaging Initiative (2017) Predictive utility of marketed volumetric software tools in subjects at risk for Alzheimer disease: Do regions outside the hippocampus matter? AJNR Am J Neuroradiol 38, 546-552. [PubMed: 28057634]

[12]. Bredesen DE, Amos EC, Canick J, Ackerley M, Raji C, Fiala M, Ahdidan J (2016) Reversal of cognitive decline in Alzheimer's disease. Aging (Albany NY) 8, 1250-1258. [PubMed: 27294343]

[13]. Chen BT, Sethi SK, Jin T, Patel SK, Ye N, Sun CL, Rockne RC, Haacke EM, Root JC, Saykin AJ, Ahles TA, Holodny AI, Prakash N, Mortimer J, Waisman J, Yuan Y, Somlo G, Li D, Yang R, Tan H, Katheria V, Morrison R, Hurria A (2018) Assessing brain volume changes in older women with breast cancer receiving adjuvant chemotherapy: A brain magnetic resonance imaging pilot study. Breast Cancer Res 20, 38. [PubMed: 29720224]

[14]. Schain M, Varnas K, Cselenyi Z, Halldin C, Farde L, Varrone A (2014) Evaluation of two automated methods for PET region of interest analysis. Neuroinformatics 12, 551-562. [PubMed: 24880728]

[15]. Lundqvist R, Lilja J, Thomas BA, Lotjonen J, Villemagne VL, Rowe CC, Thurfjell L (2013) Implementation and validation of an adaptive template registration method for ${ }^{18} \mathrm{~F}$-flutemetamol imaging data. J Nucl Med 54, 1472-1478. [PubMed: 23740104]

[16]. Gorgolewski K, Burns CD, Madison C, Clark D, Halchenko YO, Waskom ML, Ghosh SS (2011) Nipype: A flexible, lightweight and extensible neuroimaging data processing framework in python. Front Neuroinform 5, 13. [PubMed: 21897815]

[17]. Su Y, D’Angelo GM, Vlassenko AG, Zhou G, Snyder AZ, Marcus DS, Blazey TM, Christensen JJ, Vora S, Morris JC, Mintun MA, Benzinger TL (2013) Quantitative analysis of PiB-PET with FreeSurfer ROIs. PLoS One 8, e73377.

[18]. Cusack R, Vicente-Grabovetsky A, Mitchell DJ, Wild CJ, Auer T, Linke AC, Peelle JE (2014) Automatic analysis (aa): Efficient neuroimaging workflows and parallel processing using Matlab and XML. Front Neuroinform 8, 90. [PubMed: 25642185]

[19]. Tzourio-Mazoyer N, Landeau B, Papathanassiou D, Crivello F, Etard O, Delcroix N, Mazoyer B, Joliot M (2002) Automated anatomical labeling of activations in SPM using a macroscopic anatomical parcellation of the MNI MRI single-subject brain. Neuroimage 15, 273-289. [PubMed: 11771995]

[20]. Frings L, Hellwig S, Bormann T, Spehl TS, Buchert R, Meyer PT (2018) Amyloid load but not regional glucose metabolism predicts conversion to Alzheimer's dementia in a memory clinic population. Eur J Nucl Med Mol Imaging 45, 1442-1448. [PubMed: 29546632]

[21]. Landau SM, Fero A, Baker SL, Koeppe R, Mintun M, Chen K, Reiman EM, Jagust WJ (2015) Measurement of longitudinal beta-amyloid change with ${ }^{18}$ F-florbetapir PET and standardized uptake value ratios. J Nucl Med 56, 567-574. [PubMed: 25745095]

[22]. Martinez G, Vernooij RW, Fuentes Padilla P, Zamora J, Bonfill Cosp X, Flicker L (2017) ${ }^{18} \mathrm{~F}$ PET with florbetapir for the early diagnosis of Alzheimer's disease dementia and other dementias in people with mild cognitive impairment (MCI). Cochrane Database Syst Rev 11, CD012216.

[23]. Martinez G, Vernooij RW, Fuentes Padilla P, Zamora J, Flicker L, Bonfill Cosp X (2017) ${ }^{18}$ F PET with florbetaben for the early diagnosis of Alzheimer's disease dementia and other dementias in people with mild cognitive impairment (MCI). Cochrane Database Syst Rev 11, CD012883. 
[24]. Martinez G, Vernooij RW, Fuentes Padilla P, Zamora J, Flicker L, Bonfill CospX (2017) ${ }^{18}$ F PET with flutemetamol for the early diagnosis of Alzheimer's disease dementia and other dementias in people with mild cognitive impairment (MCI). Cochrane Database Syst Rev 11, CD012884.

[25]. Cummings J (2019) The National Institute on Aging-Alzheimer's Association Framework on Alzheimer's disease: Application to clinical trials. Alzheimers Dement 15, 172-178. [PubMed: 29936146]

[26]. Jack CR Jr, Bennett DA, Blennow K, Carrillo MC, Dunn B, Haeberlein SB, Holtzman DM, Jagust W, Jessen F, Karlawish J, Liu E, Molinuevo JL, Montine T, Phelps C, Rankin KP, Rowe CC, Scheltens P, Siemers E, Snyder HM, Sperling R, Contributors (2018) NIA-AA Research Framework: Toward a biological definition of Alzheimer's disease. Alzheimers Dement 14, 535562. [PubMed: 29653606]

[27]. Jack CR Jr, Wiste HJ, Weigand SD, Therneau TM, Knopman DS, Lowe V, Vemuri P, Mielke MM, Roberts RO, Machulda MM, Senjem ML, Gunter JL, Rocca WA, Petersen RC (2017) Agespecific and sex-specific prevalence of cerebral beta-amyloidosis, tauopathy, and neurodegeneration in cognitively unimpaired individuals aged 50-95 years: A cross-sectional study. Lancet Neurol 16, 435-444. [PubMed: 28456479]

[28]. Vemuri P, Lowe VJ, Knopman DS, Senjem ML, Kemp BJ, Schwarz CG, Przybelski SA, Machulda MM, Petersen RC, Jack CR Jr (2017) Tau-PET uptake: Regional variation in average SUVR and impact of amyloid deposition. Alzheimers Dement (Amst) 6, 21-30. [PubMed: 28138510]

[29]. Friedland RP, Budinger TF, Ganz E, Yano Y, Mathis CA, Koss B, Ober BA, Huesman RH, Derenzo SE (1983) Regional cerebral metabolic alterations in dementia of the Alzheimer type: Positron emission tomography with $\left[{ }^{18}\right.$ F]fluorodeoxyglucose. J Comput Assist Tomogr 7, 590598. [PubMed: 6602819]

[30]. Mosconi L, Tsui WH, De Santi S, Li J, Rusinek H, Convit A, Li Y, Boppana M, de Leon MJ (2005) Reduced hippocampal metabolism in MCI and AD: Automated FDG-PET image analysis. Neurology 64, 1860-1867. [PubMed: 15955934]

[31]. Edison P, Carter SF, Rinne JO, Gelosa G, Herholz K, Nordberg A, Brooks DJ, Hinz R (2013) Comparison of MRI based and PET template based approaches in the quantitative analysis of amyloid imaging with PIB-PET. Neuroimage 70, 423-433. [PubMed: 23261639]

[32]. Evans AC, Marrett S, Neelin P, Collins L, Worsley K, Dai W, Milot S, Meyer E, Bub D(1992) Anatomical mapping of functional activation in stereotactic coordinate space. Neuroimage 1, 4353. [PubMed: 9343556]

[33]. Landau SM, Breault C, Joshi AD, Pontecorvo M, Mathis CA, Jagust WJ, Mintun MA, Alzheimer's Disease Neuroimaging Initiative (2013) Amyloid-beta imaging with Pittsburgh compound B and florbetapir: Comparing radiotracers and quantification methods. J Nucl Med 54, 70-77. [PubMed: 23166389]

[34]. Landau SM, Mintun MA, Joshi AD, Koeppe RA, Petersen RC, Aisen PS, Weiner MW, Jagust WJ, Alzheimer's Disease Neuroimaging Initiative (2012) Amyloid deposition, hypometabolism, and longitudinal cognitive decline. Ann Neurol 72, 578-586. [PubMed: 23109153]

[35]. Aalto S, Scheinin NM, Kemppainen NM, Nagren K, Kailajarvi M, Leinonen M, Scheinin M, Rinne JO (2009) Reproducibility of automated simplified voxel-based analysis of PET amyloid ligand [11C]PIB uptake using 30-min scanning data. EurJNuclMedMol Imaging 36, 1651-1660.

[36]. Jagust WJ, Landau SM, Koeppe RA, Reiman EM, Chen K, Mathis CA, Price JC, Foster NL, Wang AY (2015) The Alzheimer's Disease Neuroimaging Initiative 2 PET Core: 2015. Alzheimers Dement 11, 757-771. [PubMed: 26194311]

[37]. Scholl M, Lockhart SN, Schonhaut DR, O’Neil JP, Janabi M, Ossenkoppele R, Baker SL, Vogel JW, Faria J, Schwimmer HD, Rabinovici GD, Jagust WJ (2016) PET imaging of tau deposition in the aging human brain. Neuron 89, 971-982. [PubMed: 26938442]

[38]. Eisenstein SA, Koller JM, Piccirillo M, Kim A, Antenor-Dorsey JA, Videen TO, Snyder AZ, Karimi M, Moerlein SM, Black KJ, Perlmutter JS, Hershey T (2012) Characterization of extrastriatal D2 in vivo specific binding of $\left[{ }^{18} \mathrm{~F}\right](\mathrm{N}$-methyl)benperidol using PET. Synapse 66 , 770-780. [PubMed: 22535514]

[39]. Savli M, Bauer A, Mitterhauser M, Ding YS, Hahn A, Kroll T, Neumeister A, Haeusler D, Ungersboeck J, Henry S, Isfahani SA, Rattay F, Wadsak W, Kasper S, Lanzenberger R (2012) 
Normative database of the serotonergic system in healthy subjects using multi-tracer PET. Neuroimage 63, 447-459. [PubMed: 22789740]

[40]. van Mourik T, Snoek L, Knapen T, Norris DG (2018) Porcupine: A visual pipeline tool for neuroimaging analysis. PLoS Comput Biol 14, e1006064.

[41]. Braak H, Alafuzoff I, Arzberger T, Kretzschmar H, Del Tredici K (2006) Staging of Alzheimer disease-associated neurofibrillary pathology using paraffin sections and immunocytochemistry. ActaNeuropathol 112,389-404.

[42]. Clark CM, Schneider JA, Bedell BJ, Beach TG, Bilker WB, Mintun MA, Pontecorvo MJ, Hefti F, Carpenter AP, Flitter ML, Krautkramer MJ, Kung HF, Coleman RE, Doraiswamy PM, Fleisher AS, Sabbagh MN, Sadowsky CH, Reiman EP, Zehntner SP, Skovronsky DM, Group AAS (2011) Use of florbetapir-PET for imaging beta-amyloid pathology. JAMA 305,275-283. [PubMed: 21245183]

[43]. Yang J, Hu C, Guo N, Dutta J, Vaina LM, Johnson KA, Sepulcre J, Fakhri GE, Li Q (2017) Partial volume correction for PET quantification and its impact on brain network in Alzheimer's disease. Sci Rep 7, 13035. [PubMed: 29026139]

[44]. Passamonti L, Vazquez Rodriguez P, Hong YT, Allinson KS, Williamson D, Borchert RJ, Sami S, Cope TE, Bevan-Jones WR, Jones PS, Arnold R, Surendranathan A, Mak E, Su L, Fryer TD, Aigbirhio FI, O’Brien JT, Rowe JB (2017) ${ }^{18} \mathrm{~F}-\mathrm{AV}-1451$ positron emissiontomography in Alzheimer's disease and progressive supranuclear palsy. Brain 140, 781-791. [PubMed: 28122879]

[45]. Choi JY, Cho H, Ahn SJ, Lee JH, Ryu YH, Lee MS, Lyoo CH (2018) Off-target ${ }^{18}$ F-AV-1451 binding in the basal ganglia correlates with age-related iron accumulation. J Nucl Med 59, $117-$ 120. [PubMed: 28775201]

[46]. Gronenschild EH, Habets P, Jacobs HI, Mengelers R, Rozendaal N, van Os J, Marcelis M (2012) The effects of FreeSurfer version, workstation type, and Macintosh operating system version on anatomical volume and cortical thickness measurements. PLoS One 7, e38234.

[47]. Saint-Aubert L, Nemmi F, Peran P, Barbeau EJ, Payoux P, Chollet F, Pariente J (2014) Comparison between PET template-based method and MRI-based method for cortical quantification of florbetapir (AV-45) uptake in vivo. Eur J Nucl Med Mol Imaging 41, 836-843. [PubMed: 24435769]

[48]. Tuszynski T, Rullmann M, Luthardt J, Butzke D, Tiepolt S, Gertz HJ, Hesse S, Seese A, Lobsien D, Sabri O, Barthel H (2016) Evaluation of software tools for automated identification of neuroanatomical structures in quantitative beta-amyloid PET imaging to diagnose Alzheimer's disease. Eur J Nucl Med Mol Imaging 43, 1077-1087. [PubMed: 26739328]

[49]. Thurfjell L, Lilja J, Lundqvist R, Buckley C, Smith A, Vandenberghe R, Sherwin P (2014) Automated quantification of ${ }^{18}$ F-flutemetamol PET activity for categorizing scans as negative or positive for brain amyloid: Concordance with visual image reads. J Nucl Med 55, 1623-1628. [PubMed: 25146124]

[50]. Dupont AC, Largeau B, Santiago Ribeiro MJ, Guilloteau D, Tronel C, Arlicot N (2017) Translocator Protein-18 kDa (TSPO) Positron emission tomography (PET) imaging and its clinical impact in neurodegenerative diseases. Int J Mol Sci 18, E785.

[51]. Largeau B, Dupont AC, Guilloteau D, Santiago-Ribeiro MJ, Arlicot N (2017) TSPO PET imaging: From microglial activation to peripheral sterile inflammatory diseases? Contrast Media Mol Imaging 2017, 6592139.

[52]. Selvaraj S, Bloomfield PS, Cao B, Veronese M, Turkheimer F, Howes OD (2018) Brain TSPO imaging and gray matter volume in schizophrenia patients and in people at ultra high riskofpsychosis: An [11C]PBR28 study. SchizophrRes 195, 206-214.

[53]. Klunk WE, Koeppe RA, Price JC, Benzinger TL, Devous MD Sr., Jagust WJ, Johnson KA, Mathis CA, Minhas D, Pontecorvo MJ, Rowe CC, Skovronsky DM, Mintun MA (2015) The Centiloid Project: Standardizing quantitative amyloid plaque estimation by PET. Alzheimers Dement 11, 1-15 e11-14. [PubMed: 25443857]

[54]. Lin KJ, Hsu WC, Hsiao IT, Wey SP, Jin LW, Skovronsky D, Wai YY, Chang HP, Lo CW, Yao $\mathrm{CH}$, Yen TC, Kung MP (2010) Whole-body biodistribution and brain PET imaging with $\left[{ }^{18}\right.$ F $]$ AV-45, a novel amyloid imaging agent-a pilot study. Nucl Med Biol 37, 497-508. [PubMed: 20447562] 


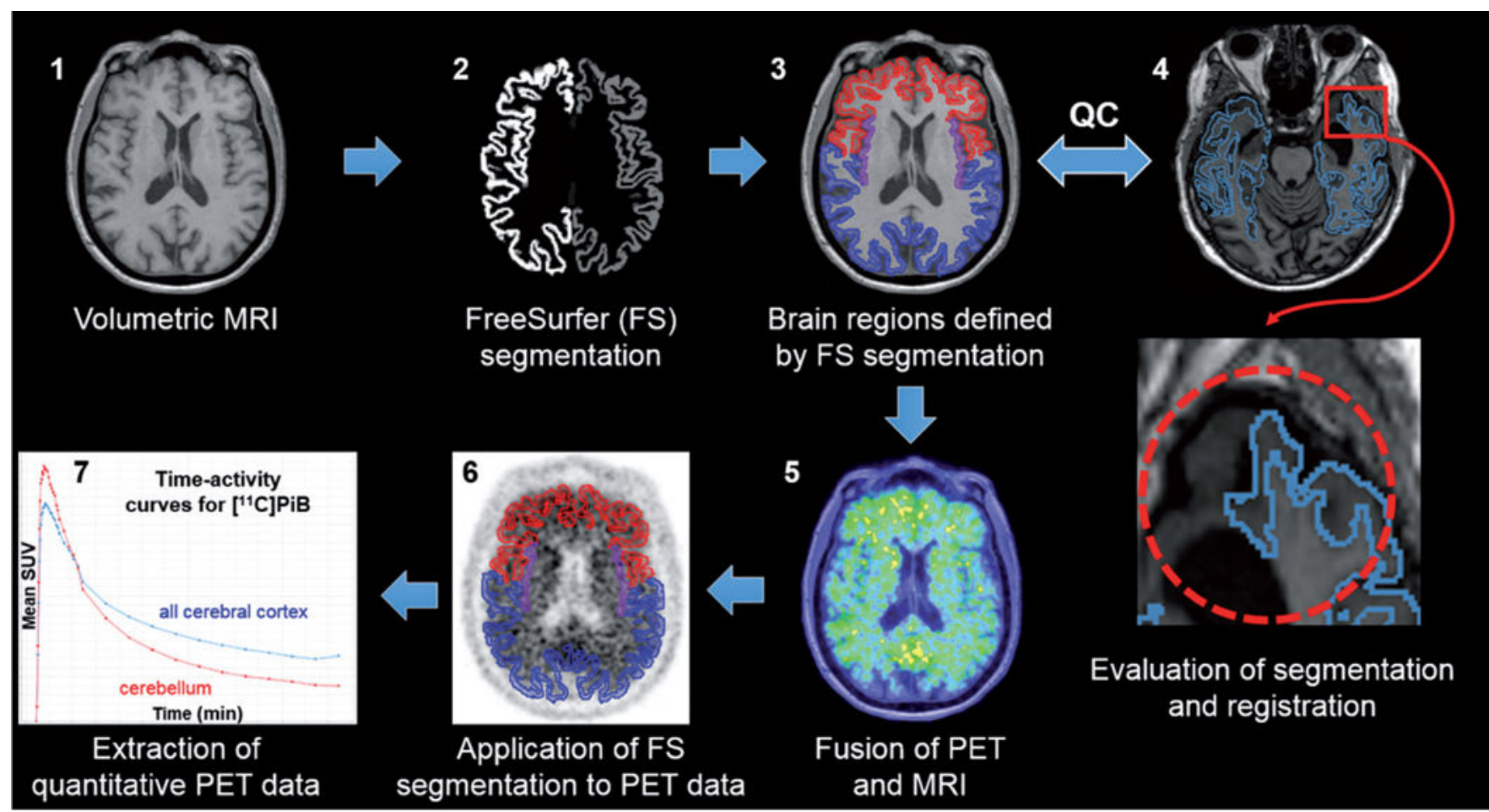

Fig. 1.

Schematic diagram of key steps in the semi-automated BLAzER methodology. The 3D volumetric MRI (1) is used to generate the Freesurfer (FS, shown in this figure) or Neuroreader (NR) segmentation (2) in DICOM format. Brain regions defined by pixel values in the FS segmentation map are generated in MIM and applied to the original MRI (3). Quality control is performed (4) to ensure both registration and segmentation are correct. In an example from a different research subject, a cortical region in the anteromedial left temporal lobe is not included in the FS-defined segmentation (red circle showing left temporal cortex not outlined by blue line). Once any errors are corrected, the PET and MRI data are fused (5). The FS-defined regions are transferred to the PET data set (6) which are then used to extract static or dynamic regional PET data (7). 

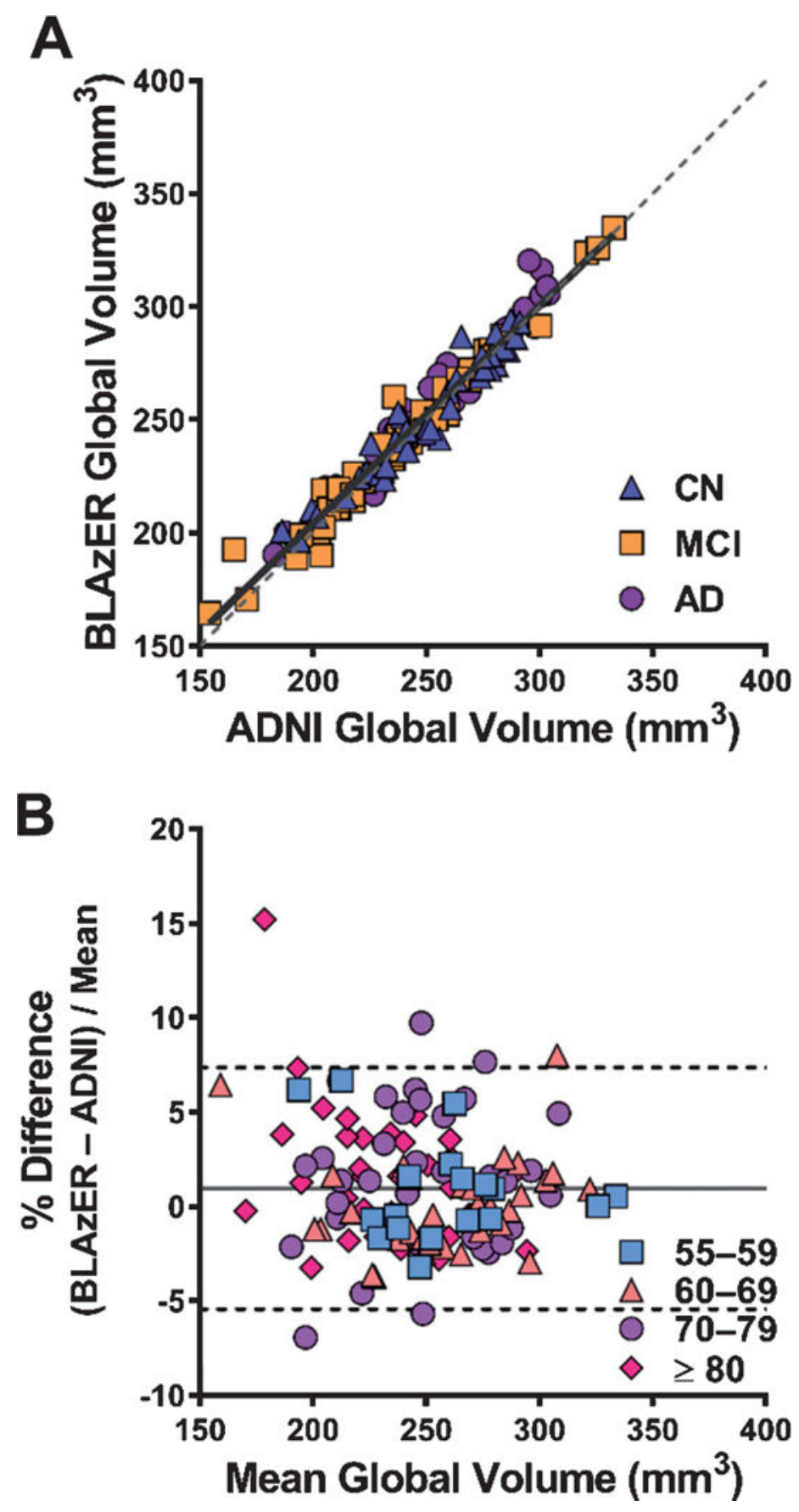

Fig. 2.

Comparison of MRI cortical volumes determined by BLAzER-FS versus ADNI. Global cerebral cortical volumes are based on frontal, temporal, parietal, and cingulate cortices as defined by the UC Berkeley analysis in ADNI. A) Univariate Pearson correlation, with regression line ( $r=0.97$, solid black), identity line (dotted), and cases coded by group. B) Bland-Altman plots with mean percent difference $(0.98 \%$, solid line) and $95 \%$ confidence intervals ([-5.43 to 7.38$]$, dotted lines), coded by age cohort. 

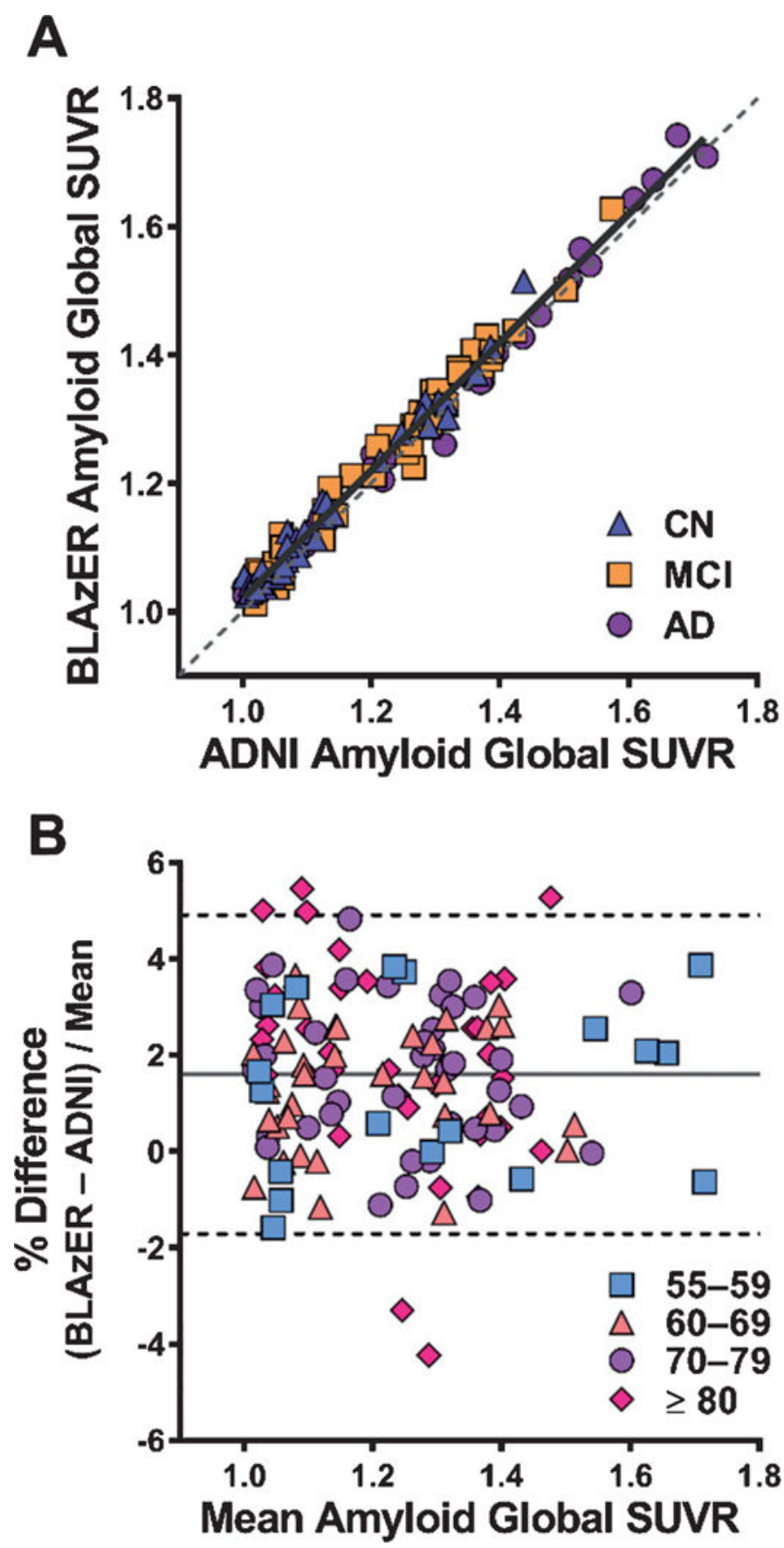

Fig. 3.

Comparison of florbetapir-PET global cerebral cortical SUVR determined by BLAzER-FS versus ADNI. A) Univariate Pearson correlation with regression line ( $\mathrm{r}=0.99$, solid black), identity line (dotted), and cases coded by group. B) Bland-Altman plots with mean percent difference (1.61\%, solid line) and $95 \%$ confidence intervals ([-1.70\% to $4.92 \%]$, dotted lines), coded by age cohort. 
A
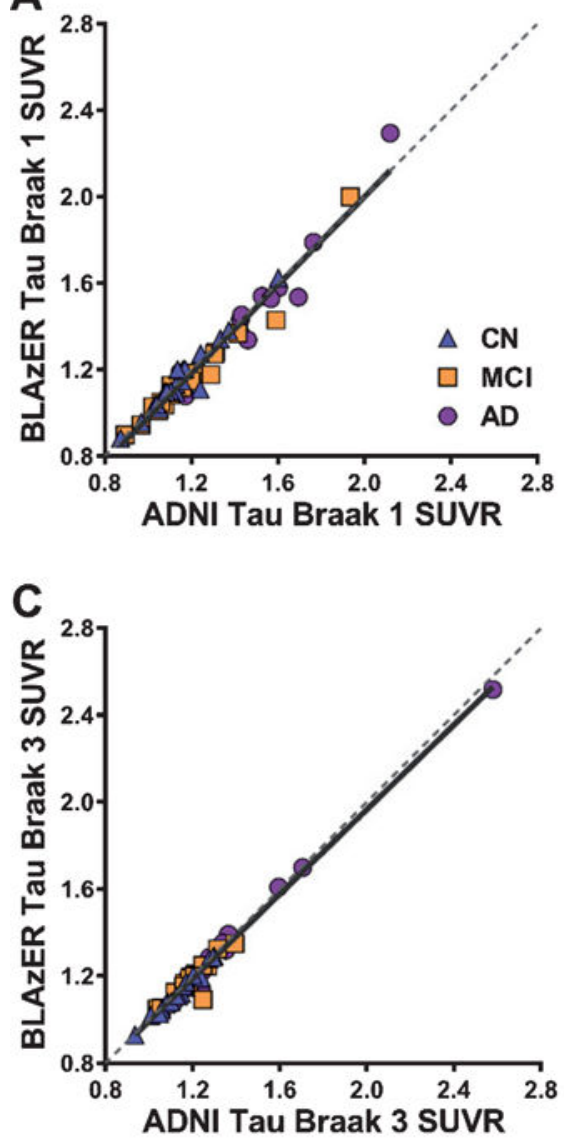

E

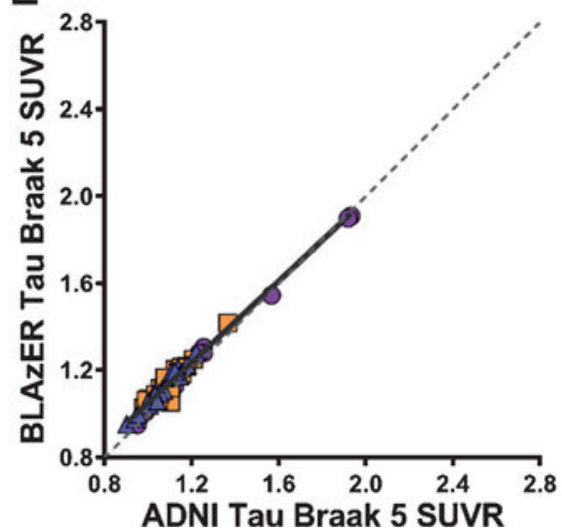

B

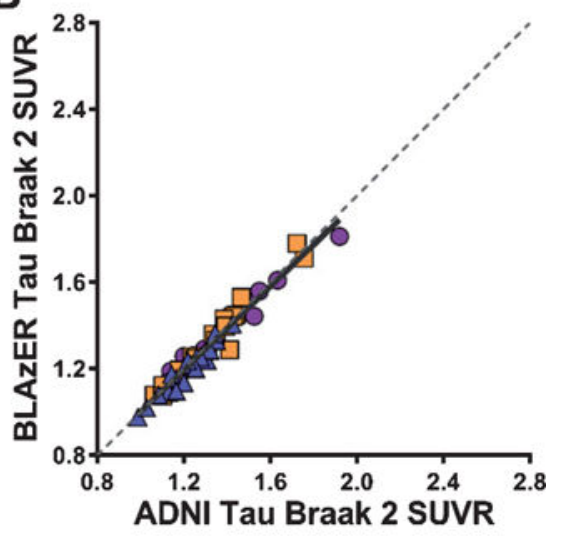

D

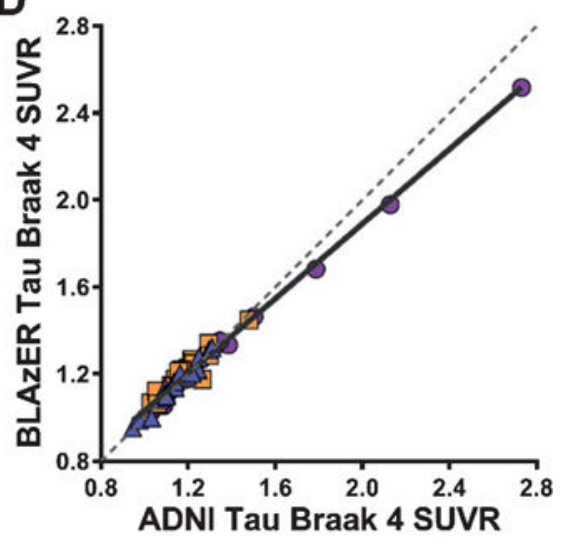

$\mathbf{F}$

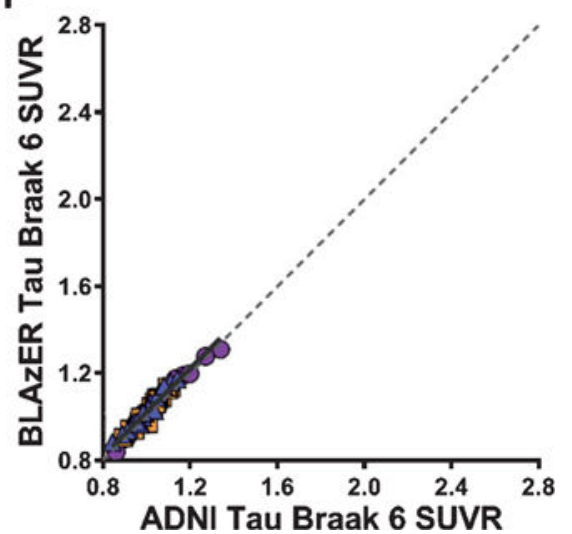

Fig. 4.

Comparison of flortaucipir-PET measured by BLAzER-FS versus ADNI across the regions representing the pathological Braak stages. (A) through (F) represent the regions for Braak stages 1 thru 6, respectively. See Methods for details on which anatomic regions were included in each stage. Univariate Pearson correlation with regression line (solid black), identity line (dotted), and cases coded by group. 

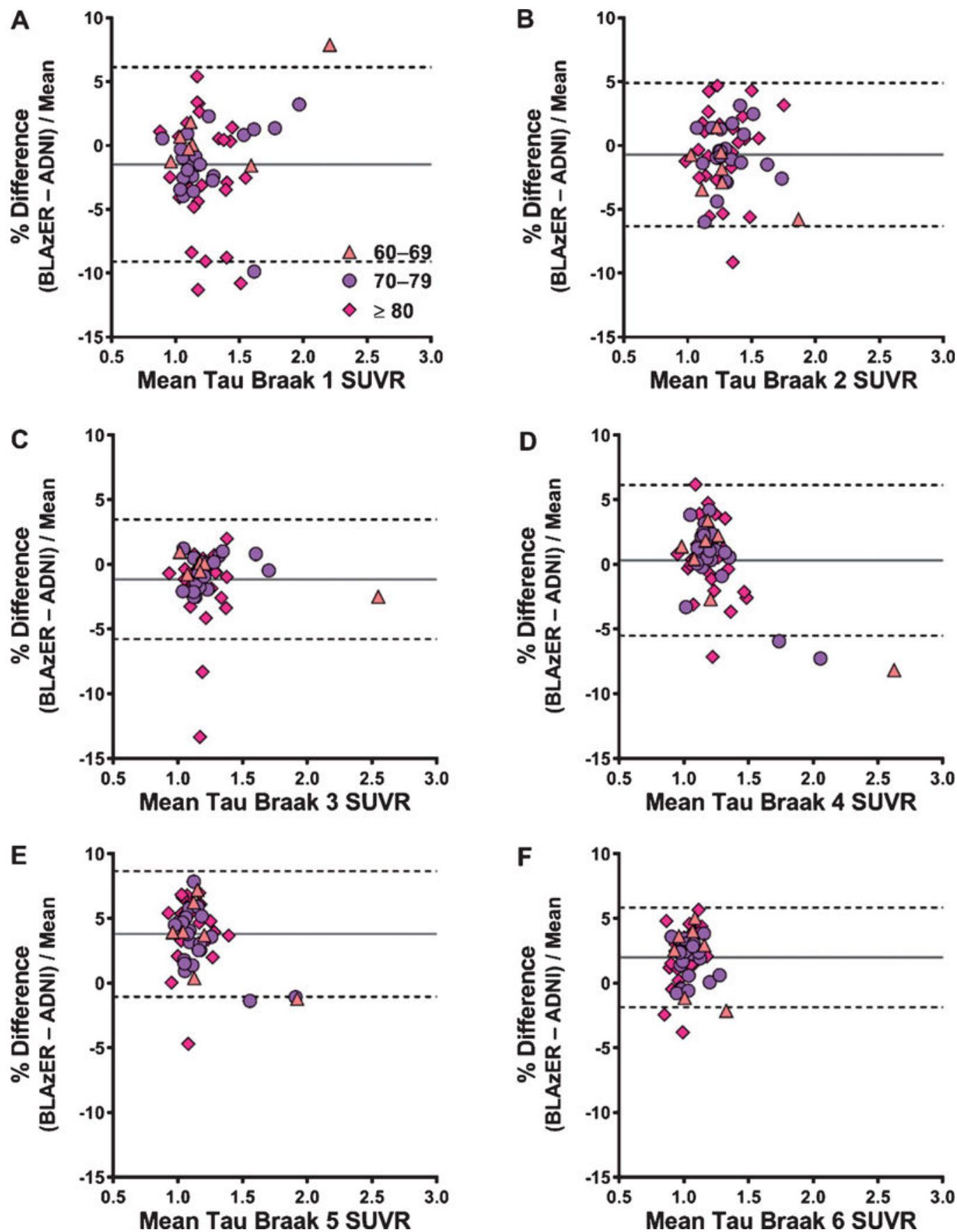

Fig. 5.

Comparison of flortaucipir-PET measured by BLAzER-FS versus ADNI across the regions representing the pathological Braak stages. (A) through (F) represent the regions for Braak stages 1 thru 6, respectively (see Methods for details on which anatomic regions were included in each stage), Bland Altman plots with mean percent difference (solid line) and $95 \%$ confidence intervals (dotted lines), coded by age cohort. 

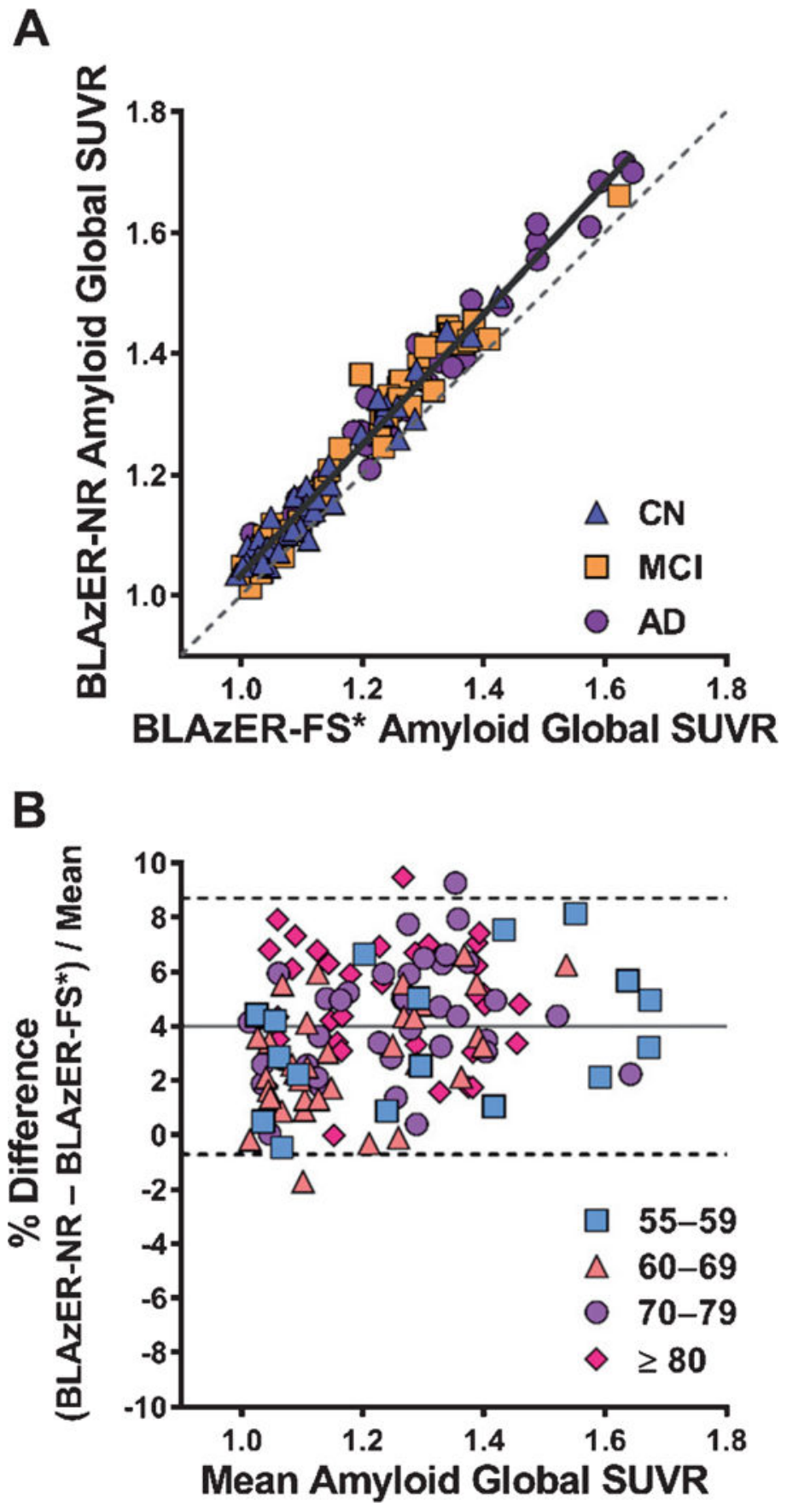

Fig. 6.

Comparison of global amyloid PET SUVR for Neuroreader (BLAzER-NR) versus FreeSurfer (BLAzER-FS*). A) Univariate Pearson correlation with regression line $(r=0.98$, solid black), identity line (dotted), and cases coded by group. B) Bland-Altman plots with mean percent difference $(4.01 \%$, solid line) and $95 \%$ confidence intervals ([ $-0.70 \%$ to $8.72 \%$ ], dotted lines), coded by age cohort. 


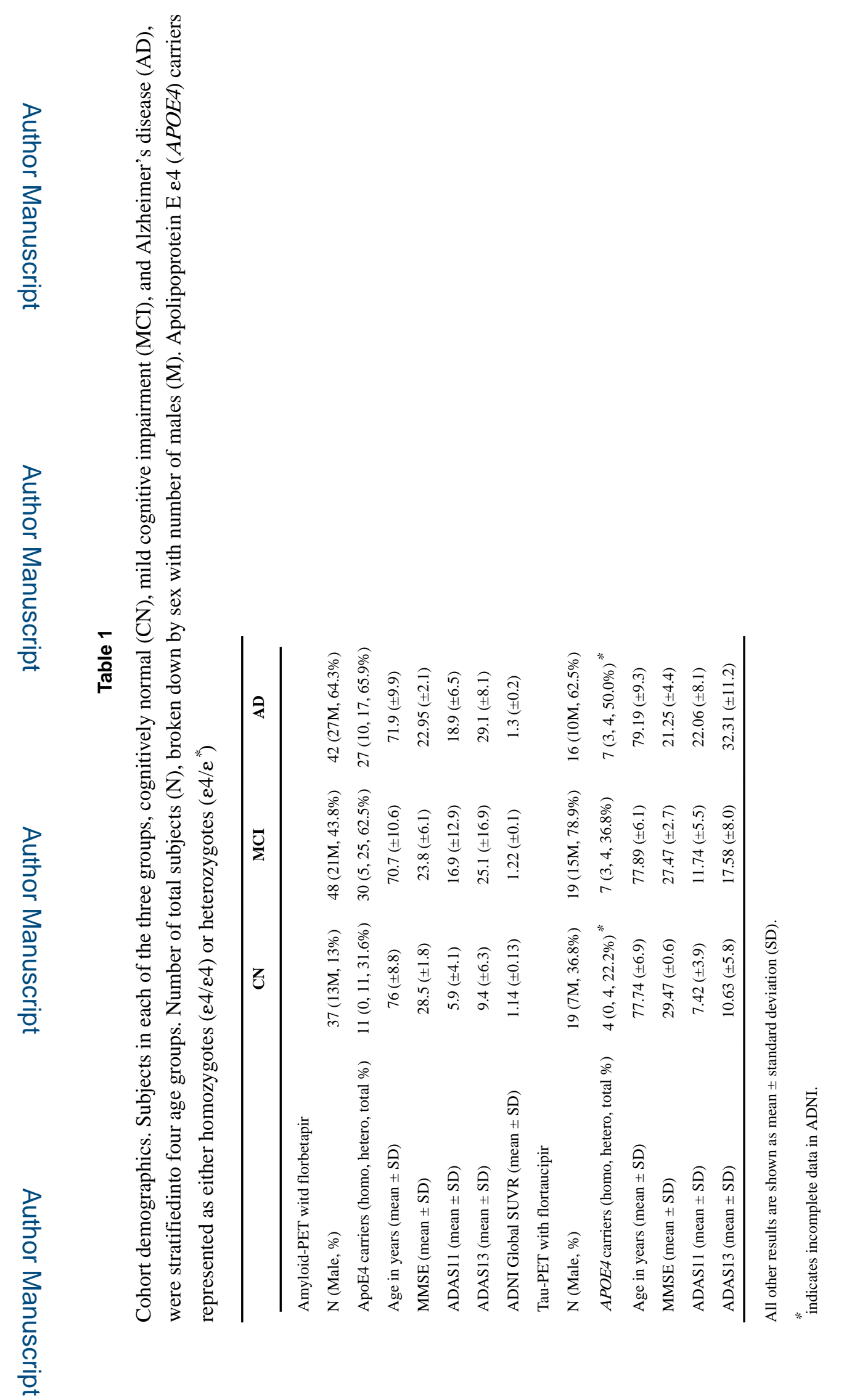

J Alzheimers Dis. Author manuscript; available in PMC 2019 September 15. 
Table 2

Key differences in post-processing methods summarized between BLAzER and the ADNI reference standard

\begin{tabular}{ll}
\hline BLAzER-FS & ADNI \\
\hline Segmentation using FS v6.0.0 & Segmentation using FS v5.3.0 \\
$\begin{array}{l}\text { One MPRAGE scan utilized for segmentation } \\
\text { Registration performed by MIM }\end{array}$ & Two independent MPRAGE scans utilized for segmentation \\
$\begin{array}{l}\text { Visualization step for quality control to ensure adequate registration and } \\
\text { manual/automated editing if necessary }\end{array}$ & Registration performed by SPM \\
$\begin{array}{l}\text { Dichotomized data both using ADNI's 1.11 cutoff as well as performing } \\
\text { regression to determine corresponding "BLAzER" units }\end{array}$ & $\begin{array}{l}\text { Derived cutoff autopsy studies of Clark et al. (34). Converted } \\
\text { their 1.10 cutoff to 1.11 "ADNI" units based on regression } \\
\text { analysis }\end{array}$ \\
\hline
\end{tabular}




\section{Table 3}

Inter-operator reproducibility of BLAzER. Interclass coefficient (ICC) values show excellent reproducibility of BLAZeR analyses between two different operators

\begin{tabular}{lcc}
\hline Region & florbetapir-PET & Volumetric MR \\
\hline Frontal & 0.9975 & 0.9914 \\
Parietal & 0.9915 & 0.9961 \\
Temporal & 0.997 & 0.9944 \\
Cingulate & 0.9961 & 0.9713 \\
Global & 0.9974 & 0.9986 \\
Region & flortaucipir-PET & \\
Braak 1 & 0.9847 & \\
Braak 2 & 0.9833 & \\
Braak 3 & 0.9963 & \\
Braak 4 & 0.9993 & \\
Braak 5 & 0.9924 & \\
Braak 6 & 0.9679 & \\
\hline
\end{tabular}




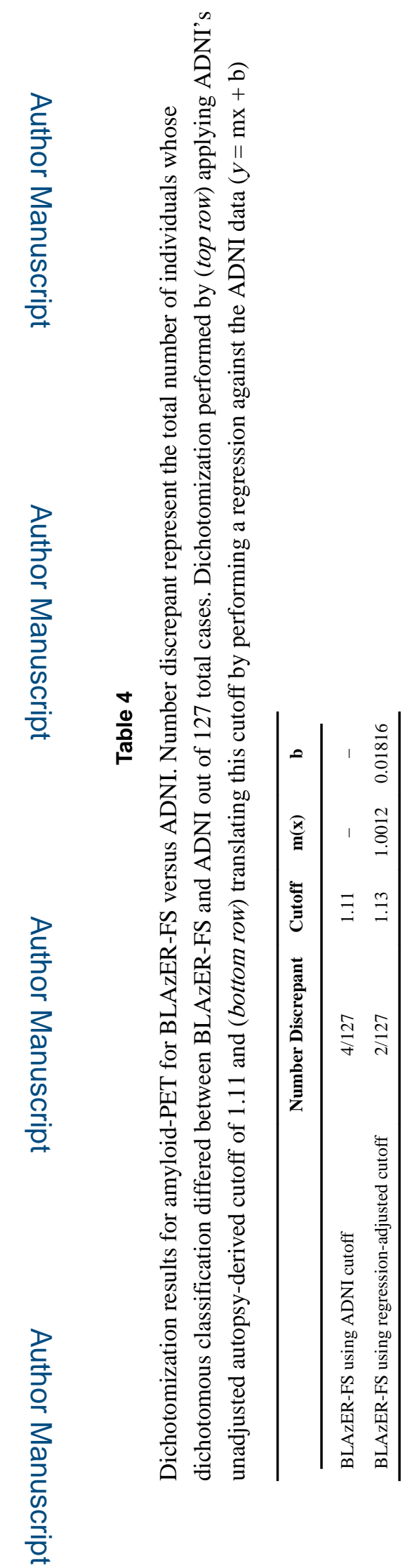

J Alzheimers Dis. Author manuscript; available in PMC 2019 September 15. 


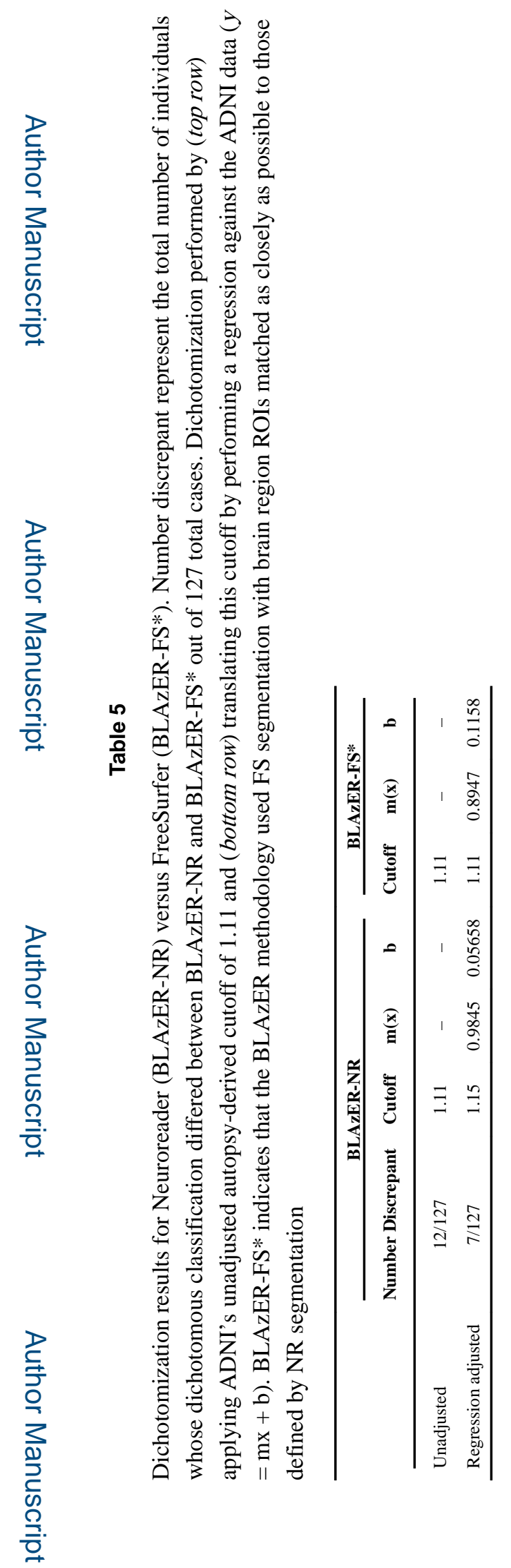

J Alzheimers Dis. Author manuscript; available in PMC 2019 September 15. 